\title{
CAPÍTULO III ESTUDIO PROSPECTIVO DEL SECTOR TECNO ECONÓMICO DE LA REGIÓN CENTRO NORTE COSTERA DEL ESTADO FALCÓN. VENEZUELA (2018-2032)
}

\author{
Mayte Josefina Maldonado Leal \\ Licda. Administración. UNERMB. Especialista en Contaduría Pública UCLA. \\ Magister en Recursos Humanos UNERMB. Dra. en Ciencias Gerenciales UNEFA. \\ Docente Universidad Nacional Experimental "Francisco Miranda" UNEFM. Email. \\ mjm10402@gmail.com.
}

\section{Nahir Minerva Rovero Sánchez}

Licda. Administración Comercial. Magister en Gerencia de Calidad y Productividad UNEFM. Doctorante en la UNEFA Ciencias Gerenciales. Docente Universidad Politécnica Territorial Alonso Gamero UPTAG. Email.nahrovero@hotmail.com.

\section{Resumen}

La investigación versa sobre un estudio prospectivo, cuyo objetivo principal consistió en diseñar los escenarios que impulsen el sector tecno económico de la región centro norte costera del estado Falcón, para ello se identificaron las variables clave, reconocieron los actores, formularon las hipótesis y esbozaron los escenarios futuros. Metodológicamente, el estudio se basó en el enfoque prospectivo de Michel Godet, empleando el MIC-MAC, la construcción de escenarios y se aplicó el juego de actores con el método MACTOR, donde se pudo conocer los actores y la correlación de fuerzas. Posteriormente, se diseñaron los escenarios futuribles, partiendo de un conjunto de hipótesis. Los resultados obtenidos evidencian que, en la región intervienen múltiples factores, actores y situaciones que inciden en el sector tecno económico, constituyendo un reto para los diferentes actores que hacen vida en la zona. Como reflexión final, se desprende que los integrantes del sector tecno económico tienen el reto y compromiso de impulsar un nuevo modelo de crecimiento económico y tecnológico, centrado en el uso racional de los recursos y la preservación del medio ambiente.

Palabras Clave: Prospectiva, región centro norte costera, sector tecno económico.

\section{PROSPECTIVE STUDY OF THE TECNO ECONÓMICO SECTOR OF THE CENTRAL NORTH COASTAL REGION OF THE STATE FALCÓN. VENEZUELA (2018-2032)}

\begin{abstract}
The research is about a prospective study, whose main objective was to design the scenarios that drive the techno-economic sector of the north-central coastal region of the Falcón state, for which the key variables were identified, the actors recognized, the
\end{abstract}


hypotheses formulated and the future scenarios. Methodologically, the study was based on the prospective approach of Michel Godet, using the MIC-MAC, the construction of scenarios and the game of actors was applied with the MACTOR method, where the actors and the correlation of forces could be known. Subsequently, the future scenarios were designed, based on a set of hypotheses. The results obtained show that, in the region, multiple factors, actors and situations that affect the techno-economic sector intervene, constituting a challenge for the different actors that make life in the area. As a final reflection, it is clear that the members of the techno-economic sector have the challenge and commitment to promote a new model of economic and technological growth, focused on the rational use of resources and the preservation of the environment.

Keywords: Prospective, north central coast region, techno economic sector.

\section{Capítulo resultado de un proyecto de investigación culminado.}

\section{Introducción}

Este capítulo, versa sobre un estudio prospectivo del sector tecno económico de la región centro norte costera del estado Falcón, en un horizonte de catorce años, el cual fue realizado en varias etapas, efectuando en primera instancia el estado del arte del sector, partiendo de investigaciones bibliográficas y llevando a cabo un trabajo de campo, pues, se entrevistaron a los actores de esta importante localidad venezolana. En segundo lugar, se procedió a la identificación de las variables clave, luego se implementó el software del MICMAC y MACTOR, finalmente se diseñaron los escenarios, cuyo resultado fue la descripción de un conjunto de situaciones futuras para esta región.

El objetivo principal de la investigación consistió en diseñar los escenarios que impulsen al sector tecno económico de la región centro norte costera del estado Falcón, el cual pudiese guiar los esfuerzos de los actores que conforman las fuerzas económicas, sociales, políticas, culturales, entre otros. Por otra parte, se generó un aprendizaje a nivel personal y profesional, por cuanto amplía nuestra visión sobre la prospectiva y sus aportes. Bajo esta perspectiva, surge el reto de explorar, considerar y trabajar en la construcción de un mejor porvenir, en medio de tantas turbulencias económicas, políticas y sociales.

\section{Identificación de las variables clave}

Antes de proceder a la identificación de las variables, resulta propicio clarificar el significado del sector tecno económico, por cuanto constituye un aspecto relevante de la investigación. En tal sentido, tenemos que este sector involucra aquellas actividades de naturaleza productiva y tecnológica asociadas a la producción que, contribuyen con el desarrollo de la zona centro norte costera del estado Falcón, la misma está conformada por los municipios Miranda, Colina, Zamora, Píritu y Tocópero.

Dentro de este contexto, conviene mencionar que los reconocimientos de las variables se derivaron del estado del arte, puesto que contiene la información principal de la zona 
en cuestión, entre éstas se encuentran: Voluntad Política (VP), Recursos financieros (RF), Desarrollo Tecnológico (DT), Diversificación Económica (DE), Marco Jurídico (MJ), Formación Especializada (FE), Productividad (P), Seguimiento y Control (SYC), Recursos Naturales (RN) y Transferencia Tecnológica (TT). De igual manera, se realizó una ponderación para cada una de las variables, visualizadas en el cuadro 1.

\section{Cuadro 1. Resultado de la calificación asignada por expertos}

\begin{tabular}{cccccccccccc}
\hline & VP & RF & DT & DE & MJ & FE & P & SyC & RN & TT & TOTAL \\
\hline VP & 0 & 4 & 4 & 4 & 2 & 4 & 3 & 4 & 2 & 4 & 31 \\
\hline RF & 1 & 0 & 4 & 3 & 2 & 3 & 3 & 2 & 2 & 4 & 24 \\
\hline DT & 1 & 1 & 0 & 4 & 1 & 2 & 4 & 2 & 3 & 2 & 20 \\
\hline DE & 1 & 3 & 2 & 0 & 1 & 2 & 3 & 2 & 1 & 3 & 18 \\
\hline MJ & 2 & 1 & 2 & 2 & 0 & 3 & 1 & 4 & 2 & 2 & 19 \\
\hline FE & 1 & 2 & 3 & 2 & 1 & 0 & 4 & 4 & 2 & 4 & 23 \\
\hline P & 1 & 4 & 3 & 2 & 1 & 2 & 0 & 1 & 2 & 1 & 17 \\
\hline SyC & 2 & 3 & 2 & 2 & 1 & 1 & 2 & 0 & 1 & 1 & 15 \\
\hline RN & 1 & 3 & 1 & 3 & 2 & 2 & 2 & 1 & 0 & 1 & 16 \\
\hline TT & 1 & 2 & 4 & 3 & 1 & 2 & 3 & 1 & 1 & 0 & 18 \\
\hline TOTAL & 11 & 23 & 25 & 25 & 12 & 21 & 25 & 21 & 16 & 22 & 0 \\
\hline
\end{tabular}

Fuente: Elaboración propia (2018).

En el cuadro anterior se expresan los resultados de la calificación asignada por los expertos, la cual fue asignada de este modo: 4: Potencialmente fuerte, 3: Moderada influencia, 2: Influyente, 1: Poco Influyente, 0: Influencia nula. En tal sentido, se visualiza que la mayor puntuación es asignada a la variable voluntad política, por considerársele uno de los elementos de mayor influencia en el sector tecno económico de la región centro norte costera del estado Falcón.

\section{Aplicación del método del MIC MAC}

En esta sección se emplea el análisis estructural/matrices de impacto cruzado (MICMAC), método que según Godet y Durance (2011) relaciona de forma ordenada las variables claves de un sistema con las de su entorno, con el objetivo de evaluar la influencia y dependencia de cada una, destacando las más influyentes y dependientes. En efecto, se evalúan las variables y sus relaciones, apoyándose en el juicio de los expertos. Sobre este particular, Cortez \& Garza (2011) manifiestan que el MICMAC busca analizar de manera cualitativa las relaciones entre las variables que componen un sistema. En este proceder se construyó el cuadro 2 . 
Cuadro 2. Listado de variables y definición del Panel de Expertos

\begin{tabular}{|c|c|c|c|}
\hline DIMENSIONES & VARIABLES & DEFINICIÓN & ACTOR SOCIAL \\
\hline Política & $\begin{array}{l}\text { Voluntad } \\
\text { política }\end{array}$ & $\begin{array}{c}\text { Interés del Estado por generar } \\
\text { un bienestar para toda la } \\
\text { sociedad. }\end{array}$ & $\begin{array}{c}\text { Gobernación y Alcaldía del } \\
\text { estado Falcón }\end{array}$ \\
\hline Económica & $\begin{array}{l}\text { Recursos } \\
\text { Financieros }\end{array}$ & $\begin{array}{c}\text { Disponibilidad económica } \\
\text { para efectuar inversiones } \\
\text { que generen desarrollo } \\
\text { económico. }\end{array}$ & $\begin{array}{c}\text { Empresa Privada (PYME) } \\
\text { Gobernación y Alcaldía del } \\
\text { estado Falcón }\end{array}$ \\
\hline Económica & $\begin{array}{l}\text { Diversificación } \\
\text { Económica }\end{array}$ & $\begin{array}{c}\text { Diversas actividades } \\
\text { económicas llevada a cabo } \\
\text { para impulsar el desarrollo de } \\
\text { una nación }\end{array}$ & Empresa Privada (PYME) \\
\hline Legal & Marco Jurídico & $\begin{array}{l}\text { Aspectos normativos que } \\
\text { regulan el sector tecno } \\
\text { económico en sus diferentes } \\
\text { instancias y niveles }\end{array}$ & Estado \\
\hline Educativa & $\begin{array}{l}\text { Formación } \\
\text { Especializada }\end{array}$ & $\begin{array}{l}\text { Desarrollo de competencias } \\
\text { específicas sobre cada área de } \\
\text { desempeño laboral }\end{array}$ & $\begin{array}{l}\text { UNEFM UNERMB,UNEFA, } \\
\text { UNESR, UBV, UPTAG }\end{array}$ \\
\hline Tecnológica & $\begin{array}{l}\text { Desarrollo } \\
\text { Tecnológico } \\
(\mathrm{I}+\mathrm{D}+\mathrm{I})\end{array}$ & $\begin{array}{l}\text { Relación de la investigación, } \\
\text { desarrollo e innovación } \\
\text { que permiten a una nación } \\
\text { incrementar el nivel de } \\
\text { ciencia y el desarrollo } \\
\text { tecnológico }\end{array}$ & $\begin{array}{c}\text { Ministerio Del Poder } \\
\text { Popular Para La Educación } \\
\text { Universitaria Ciencia Y } \\
\text { Tecnología, FUNDACITE } \\
\text { UNEFM, UNERMB,UNEFA, } \\
\text { UNESR, UBV, UPTAG }\end{array}$ \\
\hline Económica & Productividad & $\begin{array}{l}\text { Relación entre inversión, } \\
\text { recurso y producto }\end{array}$ & $\begin{array}{c}\text { Empresas Privadas Y } \\
\text { Públicas }\end{array}$ \\
\hline Gerencia & $\begin{array}{l}\text { Seguimiento y } \\
\text { Control }\end{array}$ & $\begin{array}{l}\text { Revisión de resultados de } \\
\text { planes y proyectos, desde } \\
\text { la eficacia, eficiencia y } \\
\text { efectividad para proponer los } \\
\text { cambios necesarios }\end{array}$ & $\begin{array}{c}\text { DIPLANDES } \\
\text { Gobernación y Alcaldía del } \\
\text { estado Falcón }\end{array}$ \\
\hline $\begin{array}{c}\text { Medio } \\
\text { Ambiente }\end{array}$ & $\begin{array}{l}\text { Recursos } \\
\text { Naturales }\end{array}$ & $\begin{array}{l}\text { Uso apropiado de las riquezas } \\
\text { naturales, paisajísticos, } \\
\text { hídricos, minera, } \\
\text { agropecuaria de la región }\end{array}$ & $\begin{array}{c}\text { MINISTERIO } \\
\text { DEL PODER } \\
\text { POPULAR PARA } \\
\text { ECOSOCIALISMO Y } \\
\text { AGUA }\end{array}$ \\
\hline
\end{tabular}

Fuente: Elaboración propia (2018).

\section{Análisis Estructural del MICMAC}

En esta fase se utilizó el enfoque prospectivo, basado en Michel Godet (2000) y la caja de herramientas, empleando principalmente el análisis estructural, aplicando el método MIC-MAC, el juego de actores con el método MACTOR y por último diseñando los 
escenarios, mediante la formulación de hipótesis y la aplicación de la fórmula n 2, en la que se plantean las combinaciones posibles de eventos con la ocurrencia de la hipótesis (valor uno) o no ocurrencia (valor cero) se obtiene aplicando la regla de la multiplicación 2 x 2 x $2 \times 2=16$ resultados.

Al realizar el análisis estructural, se deben haber identificado las variables que integran el sistema que, según Godet (2000) comprende un conjunto de elementos interrelacionados (variables/factores), cuya red de interrelaciones constituye la clave de sus dinámicas. Es importante señalar, que se trata de un proceso basado en el consenso colaborativo, en el cual se efectúa el inventario de variables, definiéndolas y atribuyendo una dimensión o categoría para cada una de ellas, alimentando al sistema MACTOR LIPSOR. Asimismo, se describen las relaciones entre variables, por ende, reconstituyen y describen la red de conexiones existentes. Además, se identifican las variables esenciales, donde valoran el grado de influencia que existe entre éstas, análisis que se hace vinculando pares de variables con una ponderación que va del « $0 »$ al « $\mathrm{P} »$.

En esta investigación, los expertos fueron seleccionados de las organizaciones públicas y privadas que, se vinculan con el sector tecno económico, a saber: Gobernación del estado Falcón (GF), Alcaldía del municipio Miranda del estado Falcón (AF), Dirección de Planificación y Desarrollo Social (DIPLANDES), Ministerio de Ecosocialismo y Aguas (MINEAS), Universidad Nacional Experimental "Francisco de Miranda" (UNEFM), Ministerio del Poder Popular para la Educación Universitaria, Ciencia y Tecnología (MPPECT), Universidad Nacional Experimental "Rafael María Baralt" (UNERMB), Fundación Nacional de Ciencia y Tecnología Falcón (FUNDACITE), Pequeña y mediana empresa (PYME), Universidad Nacional Experimental de las Fuerzas Armadas (UNEFA), Universidad Nacional Experimental “Simón Rodríguez” (UNESR), Universidad Politécnica Territorial “Alonso Gamero" (UPTAG) y la Universidad Bolivariana (UBV).

Una vez identificados los actores, se establecen las conexiones entre las variables. Cabe destacar que, la tabla, así como los gráficos, fueron procesados en el software LIPSOR MICMAC y exportados sus datos a Word, sin manipulación de sus resultados o valores procesados. En el plano de influencias y dependencias directas se pueden apreciar las variables que, de acuerdo con Godet, (2000) hacen posible la visualización de todos los elementos del sistema y su entorno, ubicándolas en un plano de influencia-dependencia. Este punto tiene por ordenada el indicador de influencia de la variable, y por abscisa su indicador de dependencia, tal como se observa en el siguiente gráfico. 


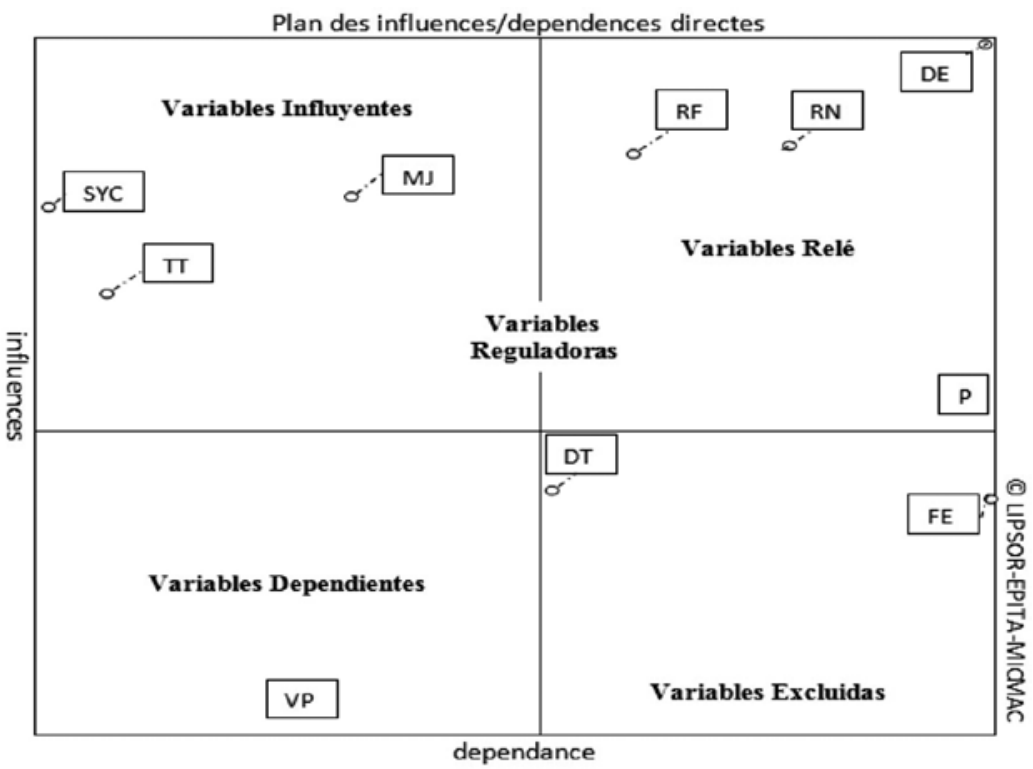

Gráfico 1. Plano de Influencias y Dependencias Directas.

Fuente: Elaboración propia (2018). Datos proporcionados por el software MICMAC*. Laboratorio LIPSOR. Prospectiker.

En el gráfico anterior se visualizan las diferentes variables que intervienen en el sistema, ejerciendo una influencia en el comportamiento del sector tecno económico. Al respecto, se presentan las Variables Influyentes, ubicadas en el cuadrante superior izquierdo, que en este caso corresponden al Seguimiento y Control, (SYC), Transferencia Tecnológica, (TT) y Marco Jurídico (MJ), las cuales actúan sobre el sistema. Además, encontramos a las Variables Relé, entendidas como factores de inestabilidad puesto que cualquier acción sobre ellas tiene consecuencias sobre los otros factores. En este cuadrante se encuentran los Recursos Financieros, (RF), Recursos Naturales, (RN), Productividad (P) y Diversificación Económica (DE).

En el gráfico también se aprecian las Variables Dependientes, que son al mismo tiempo influyentes y dependientes, por tanto, se verán afectadas por la evolución de las variables influyentes y/o las variables relé que, en este caso recayó sobre la Voluntad Política (VP). En cuanto a las Variables Excluidas, se puede decir que son al mismo tiempo poco influyentes y dependientes, se encuentran ubicadas en el cuadro inferior derecho, dentro de este cuadrante se observa la Formación especializada (FE) y Desarrollo Tecnológico (DT), éstas dependen de las decisiones de los actores. Respecto a ellas, Espinoza (2015) expone "su evolución se explica por los impactos provenientes de otras variables, en especial las de entrada y de enlace" (p.15). 
Finalmente, se visualiza a las Variables Reguladoras, que se sitúan en el centro de gravedad del sistema, en esta ocasión corresponde al Desarrollo Tecnológico (DT). Según Godet (2000), pueden actuar sucesivamente como variables secundarias, débiles objetivos y variables secundarias de riesgo. Estas influencias entre las variables, se pueden captar con mayor precisión en el siguiente gráfico.

Graphe des influences directes

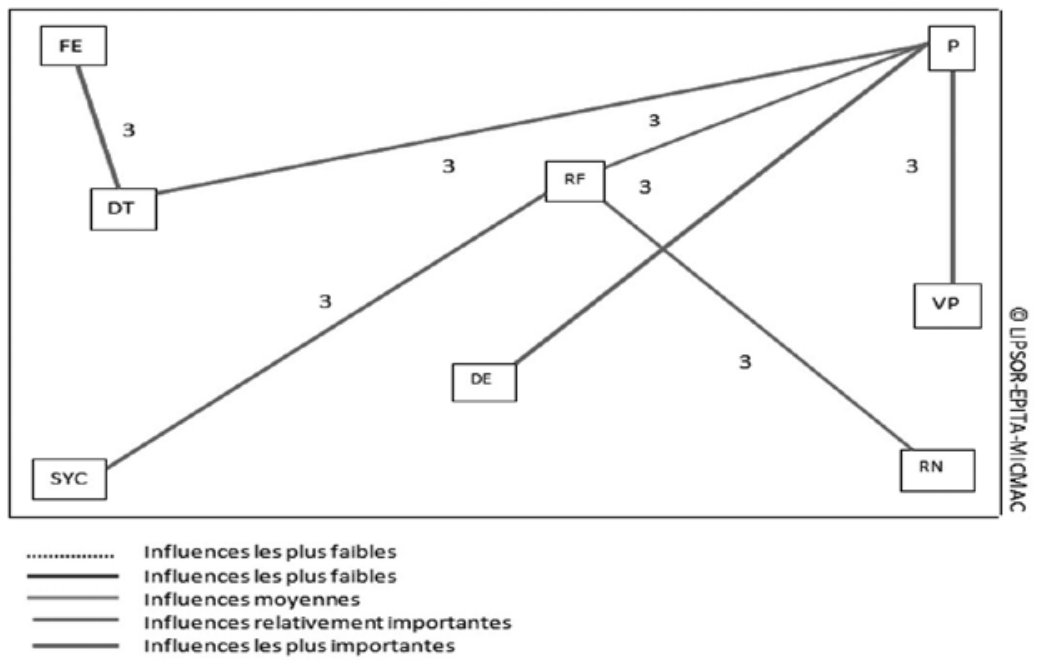

\section{Gráfico 2. Influencias Directas.}

Fuente: Elaboración propia (2018). Resultados obtenidos mediante MICMAC*. LISPSOR

En la representación anterior, se puede visualizar las Influencias directas donde intervienen las variables Voluntad Política (VP), Diversificación económica (DE) y Recursos' financieros (RF) influyen sobre la variable Productividad (P). Resulta propicio mencionar, que así como se abordan las influencias directas también se ha de valorar los planos de influencias indirectas, éstos se aprecian a continuación: 

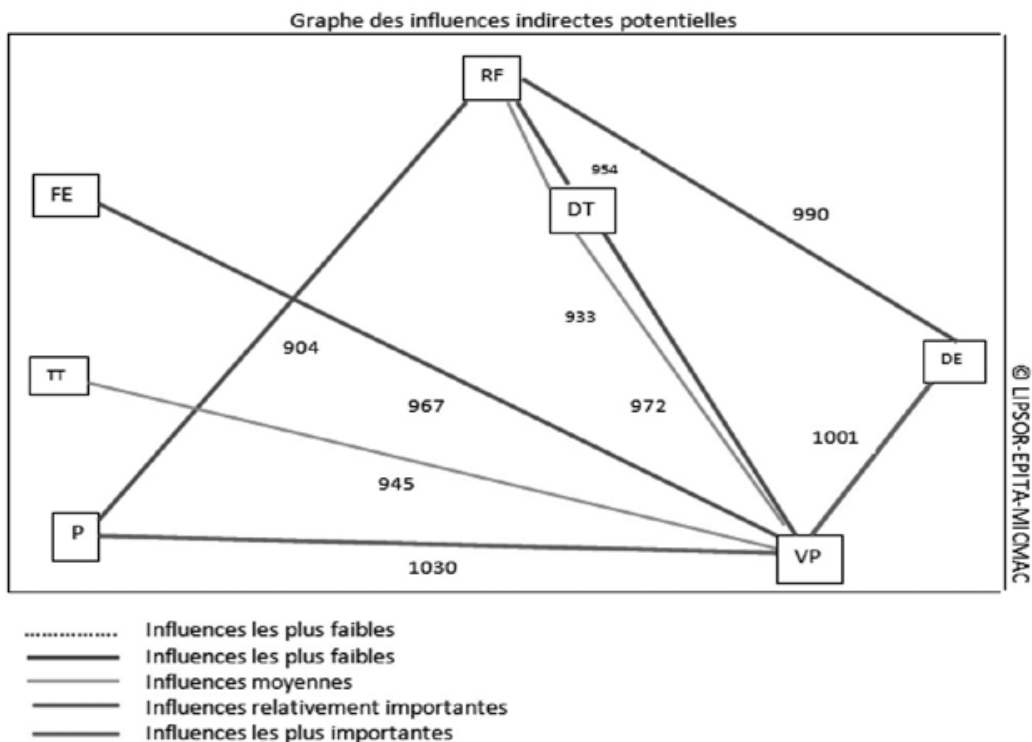

\section{Gráfico 3. Influencias Indirectas Potenciales.}

Fuente: Elaboración propia (2018). Resultados Obtenidos mediante MICMAC*. LISPSOR

El gráfico que antecede, nos permite observar la intensidad de la influencia que tiene una variable sobre la otra, en correspondencia con la ubicación de las mismas dentro del plano de influencias y dependencias. Es así como el color rojo representa, la relación entre las variables con efecto más importante; en azul intenso la influencia relativamente importante y, en azul más claro la influencia moderada.

La voluntad política tiene una influencia potencial directa de gran intensidad sobre la diversificación económica y la productividad; por cuanto se trata de variables sobre las cuales los actores pueden generar cambios. Esta condición representa una oportunidad importante, ya que puede ser controlada y dependerá de las alianzas estratégicas y de la disminución de las divergencias entre los actores.

\section{Aplicación del método MACTOR}

El MACTOR es una metodología que según Godet \& Durance, (2011) establece la correlación de fuerzas entre los diferentes actores de un sistema y examina sus afinidades y divergencias, con respecto a un determinado propósito, sus siglas significan: Método de actores, objetivos y correlación de fuerzas. En este proceder, se identificó los actores del sector tecno económico, tomando como base las variables obtenidas en el estado del arte. 
Por otra parte, Espinoza (2015) manifiesta que el método de análisis de juego de actores MACTOR, busca valorar las relaciones de fuerza entre los actores y estudiar sus convergencias y divergencias con respecto a un cierto número de posturas y de objetivos asociados. En tal sentido, la utilización del MACTOR permite identificar las posibles alianzas y conflictos entre actores, lo que es pertinente para el diseño de escenarios y el alcance de objetivos. A efectos de la presente investigación, se identificaron los actores y se realizó una ficha para cada uno. De igual forma, se construyó la matriz de influencias directas, lo cual se detalla a continuación.

\section{Matriz 1. Influencias Directas Actor por Actor}

\begin{tabular}{|c|c|c|c|c|c|c|c|c|c|c|c|c|c|}
\hline MW & $\frac{\Omega}{x}$ & 3 & 莺 & $\frac{3}{\sum_{2}}$ & 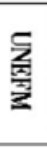 & $\frac{3}{3}$ & 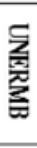 & 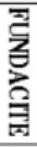 & $\underset{\Sigma}{\grave{z}}$ & 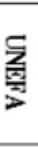 & 疍 & $\frac{\Im}{3}$ & 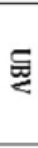 \\
\hline GF & 0 & 1 & 4 & 2 & 0 & 1 & 0 & 2 & 3 & 0 & 0 & 0 & 0 \\
\hline AM & 1 & 0 & 0 & 0 & 2 & 0 & 0 & 0 & 1 & 0 & 0 & 0 & 0 \\
\hline DIPLANDES & 2 & 0 & 0 & 2 & 0 & 0 & 0 & 0 & 1 & 0 & 0 & 0 & 0 \\
\hline MINEAS & 2 & 2 & 0 & 0 & 0 & 0 & 0 & 0 & 0 & 0 & 0 & 0 & 0 \\
\hline UNEFM & 0 & 0 & 0 & 0 & 0 & 2 & 3 & 2 & 3 & 3 & 3 & 3 & 3 \\
\hline MIPPECT & 0 & 0 & 0 & 0 & 0 & 0 & 0 & 0 & 0 & 0 & 0 & 0 & 0 \\
\hline UNERMB & 0 & 0 & 0 & 0 & 3 & 2 & 0 & 2 & 3 & 3 & 3 & 3 & 3 \\
\hline FUNDACITE & 0 & 0 & 0 & 0 & 0 & 0 & 0 & 0 & 0 & 0 & 0 & 0 & 0 \\
\hline PYME & 0 & 0 & 0 & 0 & 0 & 0 & 0 & 0 & 0 & 0 & 0 & 0 & 0 \\
\hline UNEFA & 0 & 0 & 0 & 0 & 3 & 2 & 3 & 2 & 3 & 0 & 3 & 3 & 3 \\
\hline UNESR & 0 & 0 & 0 & 0 & 3 & 2 & 3 & 2 & 3 & 3 & 0 & 3 & 3 \\
\hline UPTAG & 0 & 0 & 0 & 0 & 3 & 2 & 3 & 2 & 3 & 3 & 3 & 0 & 3 \\
\hline UBV & 0 & 0 & 0 & 0 & 3 & 2 & 3 & 2 & 3 & 3 & 3 & 3 & 0 \\
\hline
\end{tabular}

Fuente: Maldonado y Rovero (2018) Resultados Obtenidos mediante MICMAC*. LISPSOR

La matriz MID es simplemente una tabla (actores $\mathrm{x}$ actores) en donde la influencia potencial de un actor sobre el otro se registra sobre una escala de 0 a 4 . Ésta revela las relaciones de poder aparente; simplemente leyendo los totales de cada fila y cada columna. En la matriz 1 se aprecia que, la Gobernación del estado Falcón es el actor más influyente del sistema y al mismo tiempo es uno de los más susceptibles a la influencia de otros actores.

En este orden de ideas, se observa en el gráfico 5 como los actores dominados (FUNDACITE, MPPEYT,PYME) deben desarrollar estrategias para el logro de sus objetivos y converger con los actores de enlace (UNEFA, UBV, UNERMB, UNESR, UNEFM Y UPTAG) a fin de poder disminuir las divergencias en el futuro con el actor dominante (MPPEUCT), para finalmente obtener un escenario deseable que impulse y desarrolle al sector tecno económico. Por tanto, decir que un actor es dos veces más influyente que otro, en el equilibrio de poder es asignar implícitamente el doble de poder a la influencia de este actor sobre determinados objetivos. 


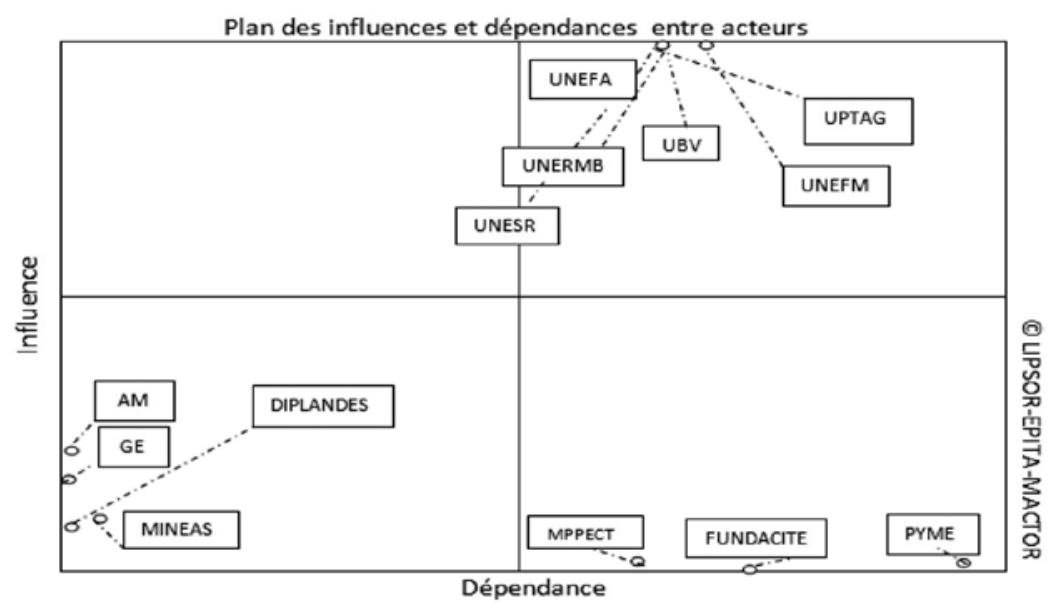

Gráfico 4. Plano de Influencias y dependencias entre actores.

Fuente: Elaboración propia (2018). Resultados Obtenidos mediante MICMAC*. LISPSOR

\section{Metodología}

La metodología se fundamentó en el enfoque de Michel Godet, empleando al respecto, el método MIC-MAC, el cual facilitó el reconocimiento de las variables que promueven al sector tecno económico y aquellas de mayor dependencia. En este análisis se tomó en consideración la información expresada por los entrevistados y la exploración bibliográfica. Asimismo, se aplicó el juego de actores con el método MACTOR, donde se pudo conocer los actores y la correlación de fuerzas. Posteriormente, se diseñaron los escenarios futuribles, partiendo de un conjunto de hipótesis.

\section{Diseño de escenarios}

En esta sección se diseñan los diferentes escenarios que, describen las múltiples opciones para determinados sucesos o hechos, detallando la situación futura y trayectoria de eventos. Al respecto Godet \& Gavaldá (1995), manifiesta que el método de escenarios tiende a construir la representación del futuro posible, así como también indica el camino para poder alcanzarlo. Vale la pena mencionar que, en estos procesos, se consideran las variables clave directas e indirectas, donde la fórmula es $\mathrm{n}^{2}$, con la ocurrencia de la hipótesis (valor uno) o no ocurrencia (valor cero), el cual se obtiene aplicando la regla de la multiplicación, 2 x 2 x 2 × $2=16$ resultados o $\mathrm{n}$ - plas posibles. Por otro lado, la construcción de escenarios se basó en la exploración de todas las combinaciones posibles de hipótesis. Éstas se detallan a continuación:

Hipótesis 1. La diversificación económica se derivará de la agricultura, pesca, artesanía, ganadería y el turismo como opciones reales de la economía de la región. 
Hipótesis 2. La voluntad política impulsará y apoyará al sector tecno económico generando beneficios a la sociedad falconiana.

Hipótesis 3. El uso racional de los recursos naturales generará el desarrollo sustentable de la región centro norte costera del estado Falcón, apoyándose en el modelo económico productivo y ecosocialista.

Hipótesis 4: El diseño e implementación de políticas que estimulen la productividad, permitirá el desarrollo competitivo y endógeno del sector tecno económico.

Cuadro 3. Tabla Probabilidad de Escenarios

\begin{tabular}{ccccc}
\hline $\begin{array}{c}\text { ESCENARIOS } \\
\left(\mathbf{E}_{\mathbf{1}} \ldots . \mathbf{E}_{\mathbf{n}}\right)\end{array}$ & $\begin{array}{c}\text { HIPÓTESIS 1 } \\
\left(\mathbf{H}_{\mathbf{1}}\right)\end{array}$ & $\begin{array}{c}\text { HIPÓTESIS 2 } \\
\left(\mathbf{H}_{\mathbf{2}}\right)\end{array}$ & $\begin{array}{c}\text { HIPÓTESIS 3 } \\
\left(\mathbf{H}_{\mathbf{3}}\right)\end{array}$ & $\begin{array}{c}\text { HIPÓTESIS 4 } \\
\left(\mathbf{H}_{\mathbf{4}}\right)\end{array}$ \\
\hline $\mathrm{E}_{1}$ & 1 & 1 & 1 & 1 \\
\hline $\mathrm{E}_{2}$ & 0 & 1 & 1 & 1 \\
\hline $\begin{array}{c}\text { ESCENARIOS } \\
(\text { E1 ... En })\end{array}$ & $\begin{array}{c}\text { HIPÓTESIS 1 } \\
(\mathrm{H} 1)\end{array}$ & $\begin{array}{c}\text { HIPÓTESIS } 2 \\
(\mathrm{H} 2)\end{array}$ & $\begin{array}{c}\text { HIPÓTESIS 3 } \\
(\mathrm{H} 3)\end{array}$ & $\begin{array}{c}\text { HIPÓTESIS 4 } \\
(\mathrm{H} 4)\end{array}$ \\
\hline E3 & 1 & 0 & 1 & 1 \\
\hline E4 & 1 & 1 & 0 & 1 \\
\hline E5 & 1 & 1 & 1 & 0 \\
\hline E6 & 0 & 0 & 1 & 1 \\
\hline E7 & 0 & 1 & 0 & 1 \\
\hline E8 & 0 & 1 & 1 & 0 \\
\hline E9 & 1 & 0 & 0 & 0 \\
\hline E10 & 1 & 0 & 0 & 1 \\
\hline E11 & 1 & 1 & 0 & 0 \\
\hline E12 & 0 & 0 & 1 & 0 \\
\hline E13 & 0 & 0 & 0 & 0 \\
\hline E14 & 0 & 1 & 0 & 0 \\
\hline E15 & 1 & 0 & 0 & \\
\hline E16 & 0 & 0 & & 0 \\
\hline
\end{tabular}

Fuente: Elaboración propia (2018).

\section{Escenarios}

A continuación, se presentan los escenarios, derivados de la combinación de hipótesis, apoyándose en los resultados obtenidos en el cuadro anterior. A saber:

Escenario 1. $\mathrm{H} 1+\mathrm{H} 2+\mathrm{H} 3+\mathrm{H} 4$. En un horizonte futurible de 14 años, la región centro norte costera del estado Falcón habrá diversificado su economía apoyándose en sus diferentes potencialidades, con el apoyo de la voluntad política al sector tecno económico, lo cual generará beneficios a la sociedad falconiana. Además, usarán racionalmente los recursos naturales, repercutiendo en el desarrollo sostenible de la región. De igual forma, 
se formularán políticas que estimulen la productividad y que permitirán alcanzar el desarrollo competitivo y tecnológico.

Escenario 2. $\mathrm{H} 2+\mathrm{H} 3+\mathrm{H} 4-\mathrm{H} 1$. Dentro de 14 años, existirá voluntad política que impulsará y apoyará al sector tecno económico generando beneficios para la sociedad falconiana, mediante el uso racional de los recursos naturales y propiciando el desarrollo sostenible. Asimismo, se formularán políticas que estimulen la productividad, a fin de alcanzar el desarrollo competitivo y tecnológico. Sin embargo, no se logrará la diversificación económica derivada de la agricultura, pesca, artesanía, ganadería y el turismo.

Escenario 3. H1+H3+H4-H2. En un lapso de 14 años, la región centro norte costera del estado Falcón habrá diversificado su economía en sus diferentes potencialidades, haciendo uso racional de los recursos naturales lo que generará el desarrollo sostenible. Además, se formularán políticas que estimulen la productividad y que facilitarán el desarrollo competitivo y tecnológico, a pesar de no contar con la voluntad política que propicie beneficios a la sociedad.

Escenario 4. H1+H2+H4-H3. Dentro de 14 años, se habrá logrado la diversificación económica como opciones reales de la economía de la región centro norte costera del estado Falcón, lo que se logrará con el apoyo de la voluntad política que, impulsará al sector tecno económico generando beneficios para la sociedad. Asimismo, diseñarán e implementarán políticas que estimulen la productividad, contribuyendo con el desarrollo competitivo y tecnológico, a pesar de que no se hará un uso racional de los recursos naturales, afectando el desarrollo sostenible.

Escenario 5. H1+H2+H3-H4. En un lapso de 14 años, en la región centro norte costera de Falcón sobrevendrá una diversificación económica derivada de la agricultura, pesca, artesanía, ganadería y el turismo como una opción real de la economía de la región, asimismo la voluntad política estará dirigida a impulsar y apoyar al sector tecno económico, mediante el uso racional de los recursos naturales hacia el desarrollo sustentable de esta región, apoyándose en un modelo económico productivo y eco socialista, pero sin la posibilidad de diseñar e implementar políticas para el estímulo de la productividad.

Escenario 6. H3+H4-H1-H2. En un futuro de 14 años en la región centro norte costera de Falcón se hará un uso racional de los recursos naturales, lo cual generará el desarrollo sustentable en la región, apoyados en un modelo económico productivo y ecosocialista, fundamentándose en el diseño e implementación de políticas para el estímulo de la productividad. Sin embargo, no se llevará a cabo la diversificación económica, tampoco se manifestará la voluntad política para el impulso del sector tecno económico.

Escenario 7. H2+H4-H1-H3. Dentro de 14 años en la región centro norte costera del estado Falcón, la voluntad política impulsará y apoyará al sector tecno económico generando beneficios para la sociedad falconiana, asimismo, los entes competentes diseñarán e implementarán políticas para el estímulo de la productividad, permitiendo alcanzar el desarrollo competitivo y endógeno, sin embargo, no se evidenciará la diversificación económica en la agricultura, la pesca, la artesanía, la ganadería y el turismo, asimismo, de ninguna manera se hará uso racional de los recursos naturales.

Escenario 8. H2+H3-H1-H4. En un horizonte de 14 años en la región centro norte costera del estado Falcón, la voluntad política impulsará y apoyará al sector tecno 
económico generando beneficios para la sociedad falconiana, además, se hará uso racional de los recursos naturales para el desarrollo sustentable de la región, apoyándose en un modelo económico productivo y eco socialista. No obstante, no se evidenciará una diversificación económica como consecuencia no se diseñarán e implementarán políticas para el estímulo a la productividad.

Escenario 9. H1-H2-H3+H4. Dentro de 14 años la región centro norte costera del estado Falcón habrá diversificado su economía, basándose en sus diferentes potencialidades, aun cuando no exista la voluntad política que apoye al sector tecno económico, pudiendo afectar negativamente a la sociedad falconiana. Adicionalmente, la zona se verá afectada por el uso inadecuado de los recursos naturales, comprometiendo su desarrollo sostenible. A pesar de que, se formulan políticas que estimulen la productividad, en aras de alcanzar el desarrollo.

Escenario 10. H1+H3-H2-H4. Dentro de 14 años, la región centro norte costera de Falcón habrá diversificado su economía, apoyándose en sus potencialidades existentes, en equilibrio con los recursos naturales disponibles, pues los usan racionalmente para satisfacer las necesidades de la población sin comprometer su futuro. Sin embargo, la voluntad política no estimulará al sector tecno económico, afectando a la sociedad falconiana. De igual manera, no se diseñarán políticas que estimulen la productividad, repercutiendo en el desarrollo competitivo y endógeno.

Escenario 11. H1+H2-H3-H4. En un período de 14 años, la región centro norte del estado Falcón habrá diversificado su economía, apoyándose en sus potencialidades, sobre la base de una voluntad política que impulsará y apoyará al sector tecno económico, generando beneficios a la sociedad falconiana. Aun cuando los recursos naturales no hayan sido usados racionalmente, comprometiendo el desarrollo sustentable, sin el apoyo de políticas que estimulen la productividad para el logro del desarrollo competitivo y endógeno.

Escenario 12. H4-H1-H2-H3. Dentro de 14 años, se formularán e implantarán políticas que estimulen la productividad, fomentando el desarrollo competitivo y endógeno de la región, aun cuando el sector empresarial tanto público como privado no diversifique su economía y, no exista una voluntad política que estimule y apoye al sector tecno económico, afectando a la sociedad falconiana. Además, no usan racionalmente los recursos naturales, repercutiendo así en el desarrollo sustentable.

Escenario 13. H3-H1-H2-H4. En un horizonte futurible de 14 años, la comunidad empleará racionalmente los recursos naturales generando su desarrollo sustentable, apoyándose al respecto en un modelo económico, productivo y eco socialista. Aun cuando, el sector empresarial tanto público como privado no diversifique su economía, ni exista una voluntad política que impulse y apoye al sector tecno económico, afectando a la región falconiana. Además, no diseñarán e implementarán políticas que estimulen la productividad, repercutiendo negativamente en el desarrollo competitivo y endógeno.

Escenario 14. H2-H1-H3-H4. En un periodo de 14 años, la voluntad política impulsará y apoyará al sector tecno económico, generando beneficios a la sociedad falconiana, a pesar de que no se diversifique la economía de la zona, ni usen racionalmente los recursos naturales, comprometiendo el desarrollo sustentable. Aunado a lo anterior, no se diseñarán 
e implantarán unas políticas adecuadas que estimulen la productividad, a los efectos de lograr el desarrollo competitivo y endógeno del sector tecno económico.

Escenario 15. H1-H2-H3-H4. En un futuro de 14 años en la región centro norte costera del estado Falcón presentará una diversificación económica en las áreas de la agricultura, pesca, artesanía, ganadería y el turismo como una opción real de la economía de la región. Sin embargo, no existirá la voluntad política que impulse y apoye al sector tecno económico, en consecuencia, no se hará uso racional de los recursos naturales lo que ocasionará el nulo desarrollo sustentable de la región, asimismo, no se apoyará en un modelo económico productivo y eco socialista, ni se desarrollarán políticas para el estímulo a la productividad.

Escenario 16. -H1-H2-H3-H4. En un horizonte de 14 años, en la región centro norte costera del estado Falcón no ofrecerá la diversificación económica en la agricultura, pesca, artesanía, ganadería y el turismo como una opción real de la economía de la región, ni tampoco la voluntad política impulsará y apoyará al sector tecno económico en la generación de beneficios para la sociedad falconiana. Por otra parte, no se procurará hacer uso racional de los recursos naturales para el desarrollo sustentable, asimismo, no se aplicará un modelo económico productivo y eco socialista ni tampoco se diseñarán e implementarán políticas para el estímulo a la productividad.

Este diseño de escenarios favoreció la reflexión sobre las diferentes opciones posibles y la interrelación que tienen los eventos. En tal sentido, se presentan las siguientes situaciones:

Escenario optimista "LA ZONA NORTE COSTERA DEL ESTADO FALCÓN, UNA REGIÓN MODELO DEL DESARROLLO SUSTENTABLE”. En este contexto se cumplen todas las hipótesis, es decir, se diversifica la economía, aprovechando las potencialidades de la región centro norte costera del estado Falcón, valiéndose de la agricultura, pesca, artesanía, ganadería y turismo, generando empleo y mejores condiciones de vida. Asimismo, la voluntad política impulsará y apoyará al sector tecno económico de la región, lo cual se traducirá en beneficios en salud, vivienda, servicios públicos, en equilibrio con la naturaleza, usando racionalmente los recursos naturales. Por otra parte, el diseño de políticas estimulará la productividad, convirtiéndose así, en una zona modelo del desarrollo sustentable.

Escenario pesimista "UN CAMINO EMPINADO". En este escenario no se cumplen ninguna de las hipótesis planteadas, por tanto, constituye un camino empinado, evidenciado en la ausencia de una diversificación económica, cerrando las posibilidades en el desarrollo de las áreas prioritarias de la región, convirtiéndose en una amenaza para las empresas. Además, no existirá una verdadera voluntad política que impulse al sector tecno económico. Por otro lado, los actores involucrados no se apoyarán en un modelo económico productivo y ecosocialista, ni utilizarán racionalmente los recursos naturales, lo cual repercutirá notablemente en la región. Aunado a lo anterior, no se diseñarán ni implementarán políticas para el estímulo a la productividad, afectando el desarrollo competitivo y endógeno.

Escenario alternativo “UNA REGIÓN EN EVOLUCIÓN”. En este escenario se presenta un avance en el cumplimiento de las hipótesis, el mismo está sujeto a transformaciones que, demanda la imperiosa necesidad de adaptarse a los cambios. Por ende, puede presentarse una moderada diversificación económica como una opción real de la economía en la región, 
apoyándose en la voluntad política, lo cual podría impulsar al sector tecno económico. Asimismo, harán uso racional de los recursos naturales, con miras a concebir el desarrollo sustentable, fundamentándose en el modelo económico productivo y ecosocialista. Finalmente, los entes competentes pudiesen diseñar e implementar políticas para el estímulo de la productividad, encaminadas naturalmente al desarrollo competitivo y endógeno.

\section{Consideraciones finales}

En el estudio prospectivo se evidenció la presencia de múltiples factores, actores y situaciones que influyen en el sector tecno económico de la región centro norte costera del estado Falcón, por tanto, se requirió una actitud activa y un pensamiento complejo por parte del equipo investigador. Es de hacer notar que, en los contextos territoriales donde se emprende la prospectiva, se muestran todo tipo de incertidumbres y emergencias, lo cual afectará directa e indirectamente las tendencias o escenarios diseñados.

Bajo este punto de vista, los actores del sector tecno económico han de asumir el reto de impulsar un nuevo modelo de crecimiento económico y tecnológico que integre todos sus componentes, generando multiplicidad de oportunidades para los conciudadanos, haciendo uso racional y equilibrado de los recursos naturales, en donde la preservación y cuidado del medio ambiente sean el norte a seguir, con miras a mejorar la calidad de vida de la generación presente y la futura. Además, la prospectiva va a brindar el enriquecimiento de la visión del futuro, mediante un proceso autocritico de reflexión, basada en la ética y valores.

\section{Referencias bibliográficas}

Cortez, D. \& Garza, J. (2011) El uso del método MICMAC y MACTOR análisis prospectivo en un área operativa para la búsqueda de la excelencia operativa a través del Lean Manufacturing. Disponible:http.www.web.facpya.uanl.mx/rev_in/ Revistas/8.2/A6.pdf. (Consulta: 2018, agosto 17).

Espinoza, R. (2015). Analizar Las Estrategias De Los Actores: El Método Mactor. Seminario Prospectiva de la Educación Superior. LUZ. Maracaibo. Venezuela.

Garza, J. \& Cortez, D. (2011) El uso del método MICMAC y MACTOR análisis prospectivo en un área operativa para la búsqueda de la excelencia operativa a través del Lean Manufacturing. Disponible: http://www.web.facpya.uanl.mx/rev_in/ Revistas/8.2/A6.pdf. (Consulta: 2018, septiembre 07).

Godet, M. \& Durance, P. (2011) La Prospectiva Estratégica para las Empresas y Territorios. Traducido por Karel García Cortina. Paris: Organización de las Naciones Unidas para la Educación, la Ciencia y la Cultura.

Godet, M. (2000). La Caja de Herramientas de la Prospectiva Estratégica. Laboratorio de Investigación Prospectiva y Estrategia. CNAM. Paris. Francia.

Godet, M \& Gavaldá J. (1995). De la anticipación a la Acción. Manual de prospectiva y estrategia. Editor Alfaomega. Barcelona España. 